Geograficando, vol. 13, n. ${ }^{\circ}$, e024, junio 2017. ISSN 2346-898X

Universidad Nacional de La Plata.

Facultad de Humanidades y Ciencias de la Educación.

Departamento de Geografía

\title{
Configuraciones del riesgo y percepción social. Los asentamientos La Lomita y Alta Tensión, Puerto Madryn, Chubut
}

\author{
Configurations of risk and social perception. La Lomita y Alta \\ Tensión settlements, Puerto Madryn, Chubut
}

\section{María Paula Ferrari}

CONICET- Instituto de Investigaciones Geográficas de la Patagonia- Universidad Nacional de la Patagonia, Argentina | mapaulaferrari@yahoo.com.ar

\section{PALABRAS CLAVE RESUMEN}

Asentamientos espontáneos

Problemáticas ambientales

Puerto Madryn

\section{KEYWORDS}

Spontaneous settlements

Environmental problems

Puerto Madryn
El trabajo analiza la problemática del riesgo en los asentamientos La Lomita y Alta Tensión de Puerto Madryn, provincia de Chubut. Para tal fin, se aborda el diagnóstico de los procesos de peligrosidad y condiciones de vulnerabilidad presentes. Asimismo, se incluye el estudio de las percepciones que los residentes de estos asentamientos construyen respecto a las situaciones de peligro y vulnerabilidad identificadas. La metodología empleada consistió en la realización de relevamientos en terreno, entrevistas en profundidad con actores clave, realización de talleres comunitarios y la aplicación de encuestas de percepción del riesgo. Las similitudes encontradas entre los resultados del diagnóstico del riesgo y de las percepciones sobre el mismo plantean un escenario propicio para el diseño consensuado de acciones de gestión de la problemática.

\footnotetext{
ABSTRACT

The paper analyzes the problem of risk in the La Lomita and Alta Tensión settlements in Puerto Madryn, Chubut province. For this purpose, the diagnosis of hazard processes and vulnerability conditions is performed. Includes the study of the perceptions that the residents of these settlements construct regarding the situations of hazard and vulnerability identified. The methodology used consisted of field surveys, in-depth interviews with key stakeholders, community workshops and the application of risk perception surveys. The similarities found between the results of the diagnosis of the risk and the perceptions about the risk present an auspicious aspect for the consensual design of management actions of the problem.
} 


\section{Introducción}

El territorio como construcción social e histórica presenta diversas problemáticas, entre las que se encuentran las configuraciones del riesgo (Cardona, 2003). Puerto Madryn, que de acuerdo con su tamaño poblacional se define como una ciudad intermedia, no está exenta de esta problemática. La ciudad se constituye en un centro urbano destacado del norte de Patagonia, donde coexisten actividades industriales, portuarias, urbano residenciales y turísticas. El crecimiento que ha experimentado en los últimos años contribuyó a extender la urbanización hacia determinados sectores no aptos para la instalación humana, debido a que en muchos de ellos actúan procesos geomorfológicos vinculados con la erosión hídrica y la remoción en masa. En este sentido, la intervención antrópica interfiere la dinámica natural -mediante la instalación de viviendas en el tope de los acantilados y en cañadones de escurrimiento- y contribuye a incrementar o inducir los procesos erosivos ya existentes en determinados sectores.

La aparición de asentamientos precarios y no planificados en las áreas periféricas se constituye en una problemática en auge para la ciudad de Puerto Madryn, debido a que en el transcurso de los últimos cinco años se han expandido aceleradamente (Ferrari, 2016), tanto en el sector noroeste, oeste y sudoeste, evidenciando el conflicto de acceso y propiedad a la tierra. Los asentamientos La Lomita y Alta Tensión son un fiel reflejo de esta problemática, a la que se le incorpora aquella vinculada a las configuraciones del riesgo a partir de la presencia -en dichos espacios- de condiciones de peligro originadas por causas naturales y peligros de tipo tecnológicos.

El objetivo del trabajo es analizar las configuraciones del riesgo en los asentamientos Alta Tensión y La Lomita de Puerto Madryn, abordando el diagnóstico de las condiciones de peligrosidad de los procesos naturales e inducidos antrópicamente, y describiendo las condiciones de vulnerabilidad de los contextos expuestos a los distintos peligros. Asimismo, se incluye el estudio de las percepciones que los residentes de estos asentamientos construyen respecto a las situaciones de riesgo analizadas, con el propósito de realizar comparaciones entre ambos resultados obtenidos, tendientes a proponer acciones de gestión.

\section{2. Área de estudio}

Los asentamientos La Lomita y Alta Tensión se localizan dentro del ejido de la ciudad de Puerto Madryn, en el sector noroeste de la misma. Pertenecen a un área de expansión no planificada denominada Nueva Chubut, la cual comprende otros asentamientos espontáneos (1). El área donde se emplazan corresponde a una zona de quebradas y hondonadas con una topografía accidentada. El sector se encuentra al pie de la barda donde la meseta plana que rodea la ciudad desciende hacia la planicie costera (ver figura 1).

El clima de la ciudad posee las características áridas de la región, atemperadas por la proximidad del mar y por estar ubicada a sotavento de la meseta patagónica. La precipitación media anual es de $160 \mathrm{~mm}$, concentrándose en los meses de mayo y junio. Este hecho, sumado a los suelos predominantemente arcillosos sobre los que se emplazan los asentamientos estudiados, plantea una problemática compleja en momentos de precipitaciones intensas. 
Figura 1. Área de asentamientos del sector noroeste de Puerto Madryn

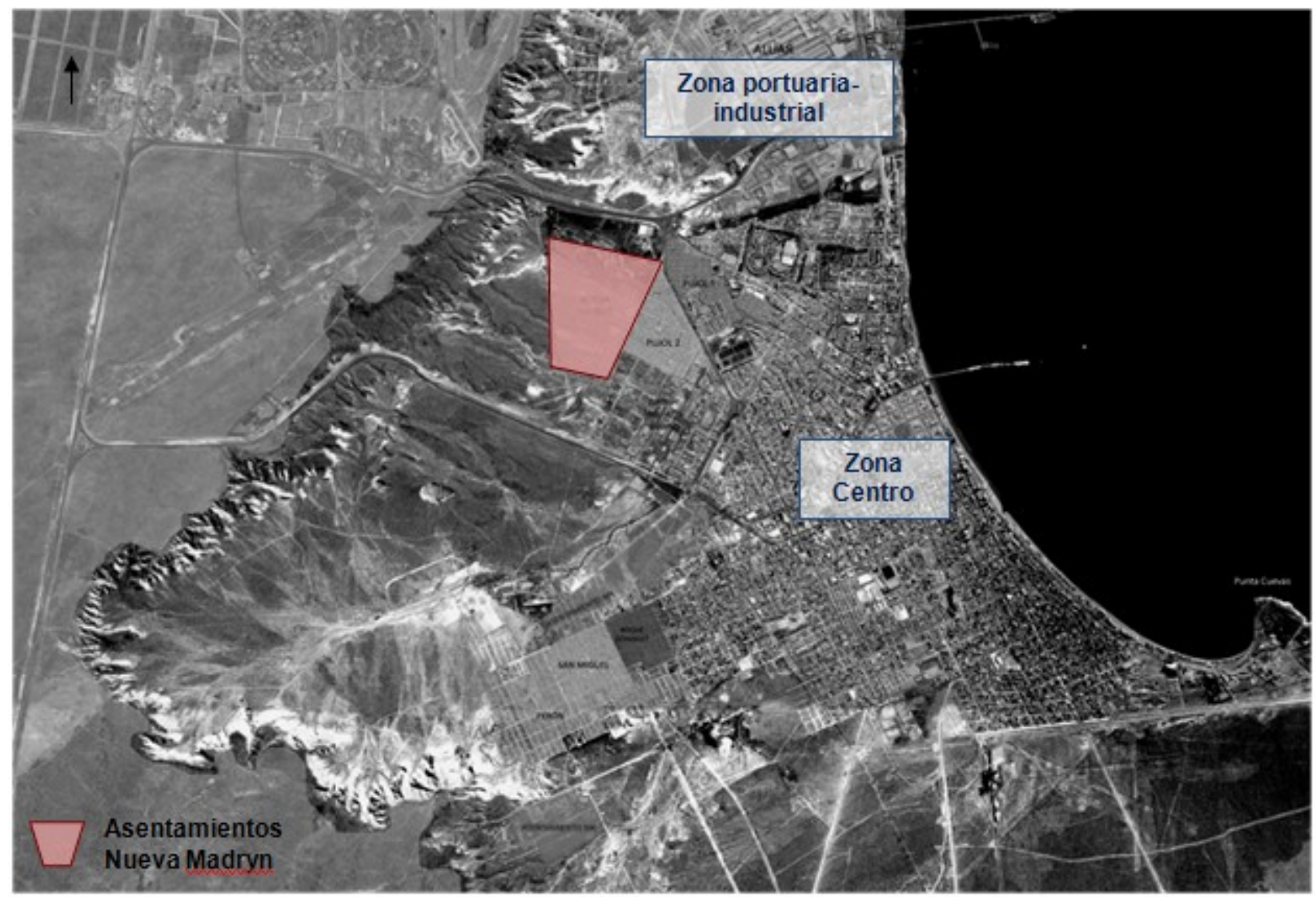

Fuente: elaboración propia a partir de Imagen Google Earth 2015

La elección de La Lomita y Alta Tensión para el abordaje de las configuraciones del riesgo se debe principalmente a las condiciones de emplazamiento descriptas, y al interés en indagar las percepciones de los residentes respecto a los motivos por los cuales eligieron asentarse allí y denominar a los asentamientos bajo estos términos (ver figura 2). 
Figura 2. La Lomita y Alta Tensión (Puerto Madryn)

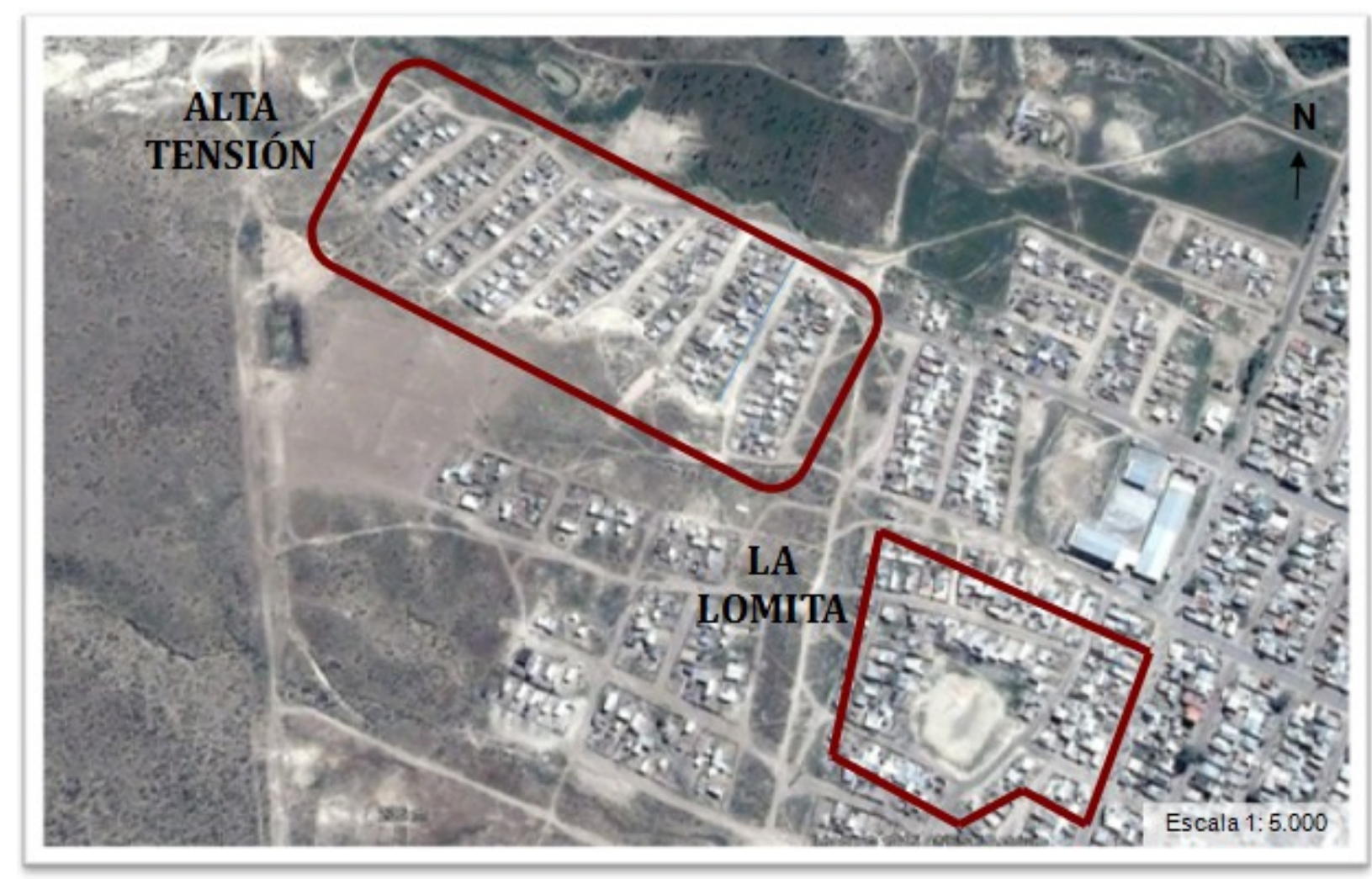

Fuente: Elaboración propia sobre imagen Google Earth 2015

Las primeras radicaciones en ambos asentamientos se realizan en el año 2008. Como expresa una de las personas entrevistadas: "agarramos un pedazo de tierra y levantamos la casa, la gente abrió las calles siguiendo el trazado de las calles circundantes.” (L.B., referente Alta Tensión). Existe una fuerte heterogeneidad respecto a la procedencia de quienes habitan los asentamientos, la mayoría proviene de la República de Bolivia y en menor medida de las provincias de Mendoza, Corrientes y Buenos Aires, así como de las localidades del interior de la provincia de Chubut.

\section{Aproximación teórica}

Los asentamientos espontáneos corresponden a aquellos sitios o lugares donde se establece una persona o comunidad al margen de los reglamentos o normas establecidas por las autoridades encargadas del ordenamiento urbano (Guerrero, 2015). Por su parte, los riesgos como problemática ambiental son el resultado de alteraciones negativas que se producen en la relación sociedadnaturaleza. Llevan impreso un carácter social y resultan de las decisiones con las cuales la sociedad construye su ambiente, provocando cambios tanto en los sistemas sociales como en los naturales, de modo que directa o indirectamente afectan la sustentabilidad del mismo. Cuando hablamos de una situación de riesgo, hacemos referencia al grado de pérdidas y/o daños esperados debido a la ocurrencia de un fenómeno peligroso desde la perspectiva humana y a la presencia de condiciones de vulnerabilidad en los elementos o contextos expuestos a dicho fenómeno (Cardona, 1993). Por lo 
tanto, el riesgo corresponde a un daño en potencia o un daño probable, y se configuran escenarios de riesgo cuando se produce la convergencia en tiempo y espacio de sus dos componentes principales: peligro y vulnerabilidad.

La peligrosidad o amenaza es considerada como la probabilidad de ocurrencia de un fenómeno natural o tecnológico, que puede presentarse en un sitio especifico y en un tiempo determinado, produciendo efectos adversos en personas, bienes o medio ambiente (Cardona, 1993). Los fenómenos de peligro que tienen su origen en procesos de la naturaleza pueden ser inducidos por intervenciones del hombre. Similar situación sucede con aquellos riesgos cuyo origen se vincula a la acción humana -por ejemplo, derrames de petróleo o accidentes nucleares- que también pueden generar peligrosidades “encadenadas” (Monti, 2011).

Desde un enfoque convencional de la geografía de los riesgos, la vulnerabilidad se entiende en términos de exposición física a un peligro. Bajo esta interpretación, la vulnerabilidad viene definida esencialmente por el fenómeno natural y no por cuestiones humanas o sociales. Existe, sin embargo, una interpretación alternativa de la vulnerabilidad -desde donde se la aborda en este trabajo- según la cual abarca un espectro más amplio que de la simple exposición física. Esta interpretación se pregunta no tanto por la severidad del agente causal, sino por otros factores (sociales, políticos, económicos o educativos) que intervienen a la hora de poner en situación de riesgo a personas o comunidades humanas. Así, entendemos por vulnerabilidad "la incapacidad de una comunidad de absorber y adaptarse a los efectos de un determinado cambio en su medio” (Wilches Chaux, 1993, p. 23). La vulnerabilidad física se refiere a la localización de asentamientos humanos en zonas de riesgo. La vulnerabilidad socioeconómica, hace referencia a las condiciones habitacionales y laborales de los grupos afectados; y la vulnerabilidad jurídica remite a las reglamentaciones y/o regulaciones relacionadas a las problemáticas de riesgos (2) $)$.

Abordar la percepción del riesgo desde las ciencias sociales "supone el estudio de las creencias, actitudes, juicios y sentimientos, así como los valores y disposiciones sociales que las personas adoptan ante los peligros” (Puy Rodríguez, 2002, p. 65). Interesa analizar las percepciones que un grupo social construye sobre las problemáticas vinculadas con los riesgos, a fin de explicar la forma en que las mismas intervienen en los comportamientos y acciones sobre el territorio. Como plantea Caneto (2000, p. 9) “si bien cada persona percibe el espacio de una manera única, el proceso de socialización determina que ciertos aspectos de las imágenes mentales sean compartidos por grandes grupos”; y desentrañar la imagen colectiva, construida a partir de imágenes y percepciones individuales, interesa a la Geografía, particularmente por esa capacidad para transformar el espacio o territorio.

\section{Metodología}

Para el abordaje de las configuraciones del riesgo se realizaron relevamientos en terreno en ambos asentamientos a fin de identificar los procesos de peligro presentes. Asimismo, se indagó bibliografía sobre el tema y estudios previos realizados desde la Municipalidad de Puerto Madryn. También se concretaron una serie de entrevistas en profundidad con actores clave, referentes de cada asentamiento y actores municipales (므). 
Por su parte, el estudio de las percepciones del riesgo se basó en la concreción de dos talleres comunitarios y la realización de encuestas personales a residentes de ambos asentamientos. Los talleres se llevaron adelante en el mes de octubre de 2015, convocados con el apoyo de los referentes de cada asentamiento. El propósito fue indagar las percepciones de los vecinos asentados respecto a sus necesidades y problemas. A los mismos asistieron aproximadamente unos 20 vecinos de ambos asentamientos, mayormente mujeres y niños. Respecto a la encuesta, la misma se diseñó con el objetivo de indagar las percepciones de las condiciones de emplazamiento y peligrosidad del sitio, así como la percepción de las condiciones de vulnerabilidad de los asentamientos (4)). Mediante la técnica bola de nieve (ㅁ) , se concretaron 60 encuestas en el asentamiento Alta Tensión y 40 encuestas en La Lomita.

\section{Las configuraciones del riesgo}

En el asentamiento Alta Tensión la instalación de viviendas se realiza en sectores mayormente planos, aunque existen viviendas emplazadas sobre laderas y próximas a cañadones de escurrimiento superficial (ver figura 3). Asimismo, el asentamiento se encuentra atravesado por la línea de media y alta tensión municipal (de ahí deriva la designación del mismo), desde donde los vecinos realizan las tomas clandestinas de electricidad para cada lote (ver figura 4). Por este motivo, las parcelas que se encuentran más próximas al tendido eléctrico deben ser relocalizadas hacia otro sector, según establecen las normas de seguridad.

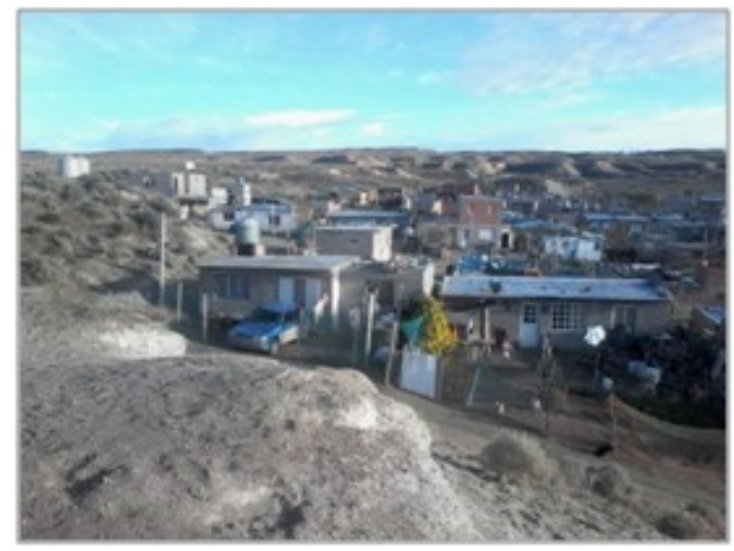

Figura 3. Viviendas sobre laderas (Alta Tensión)

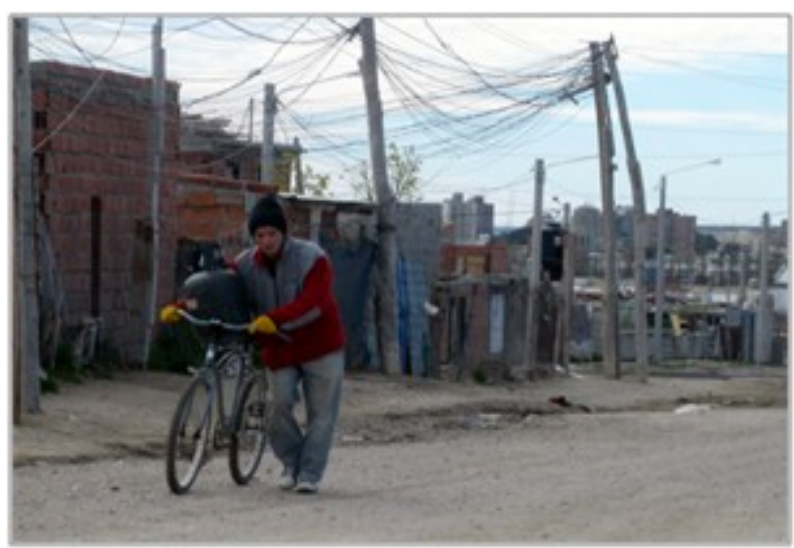

Figura 4. Tomas clandestinas de electricidad 
Por su parte, el asentamiento La Lomita se emplaza sobre un área elevada topográficamente (ver figura 5). Asimismo, en el área central del asentamiento existe un sector bajo, utilizado para actividades de esparcimiento por parte de los vecinos llamado "Bombonerita”, que en momentos de lluvias extraordinarias evidencia recurrentes inundaciones, ya que actúa como un "pozón” facilitando el deposito del agua de precipitación (ver figura 6).

\section{Figura 5.}

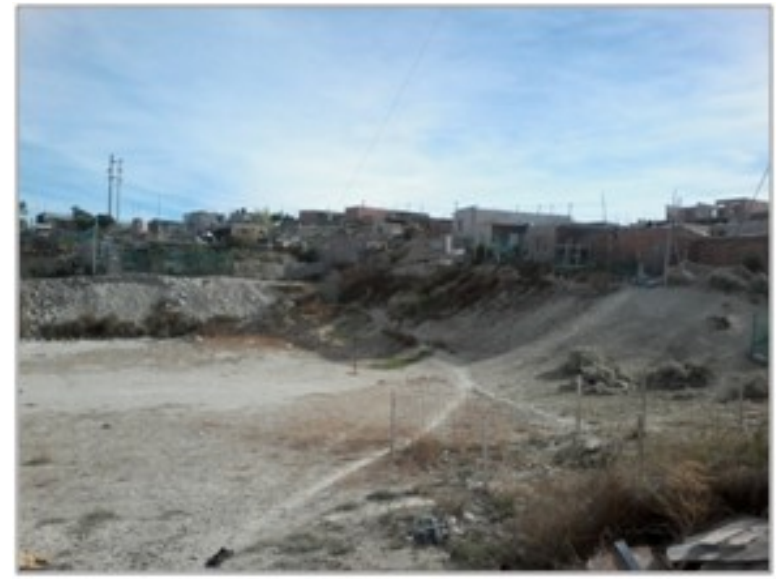

Viviendas en sector elevado "La Lomita”

Figura 6.

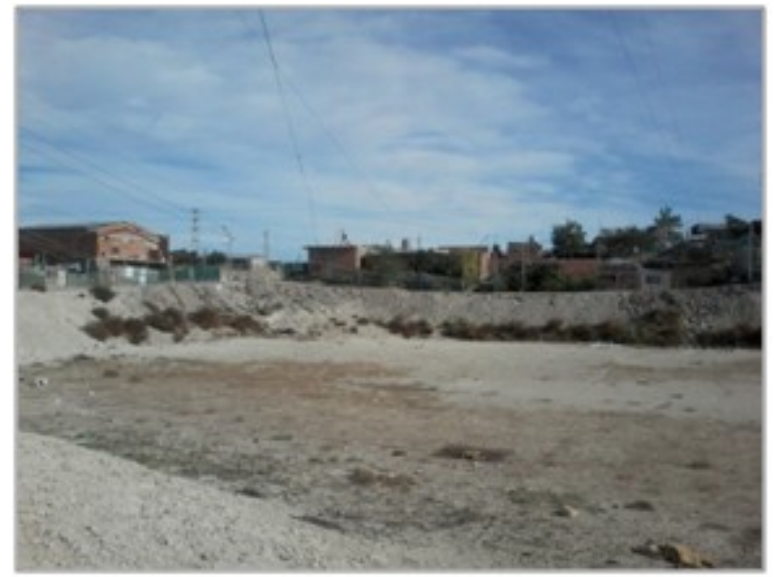

La “Bombonerita”

Teniendo en cuenta las condiciones de emplazamiento -descriptas anteriormente- se ha podido identificar la presencia de procesos de peligrosidad en los asentamientos estudiados, vinculados tanto a la dinámica natural como a las intervenciones humanas que inducen procesos de peligro.

En el caso de Alta Tensión, como puede observarse en la figura 7, los procesos de peligrosidad identificados son:

- Derrumbes por erosión hídrica: se evidencia en el sector norte del asentamiento, en contacto 
con un cañadón de escurrimiento natural. También es el caso de aquellas viviendas localizadas en el sector sur del asentamiento, sobre el frente de erosión, expuestas a probables derrumbes por acción de la erosión hídrica. Situaciones que corresponden a peligrosidades naturales inducidas antrópicamente, es decir, existen procesos de erosión hídrica natural en ambos sitios, que son acentuados por la instalación de viviendas y consiguiente modificación del sustrato.

- Inundaciones: Alta Tensión se emplaza sobre una planicie que recibe las aguas de escorrentía de las laderas que lo rodean. El sector central del mismo, se expone a eventos de inundación en momentos de precipitaciones extraordinarias. En este caso estamos hablando de un proceso de peligrosidad de tipo natural.

- Erosión hídrica: se evidencia sobre las calles que trazan el amanzanamiento. Las mismas tienen una pendiente sur-norte, que en momentos de lluvias intensas evidencian procesos de erosión por causas naturales.

- Exposición a daños por alta tensión eléctrica: corresponde a un tipo de peligrosidad tecnológica, que se encuentra presente en las parcelas ubicadas más próximas a la línea de alta tensión, en el sector noreste del asentamiento.

\section{Figura 7}

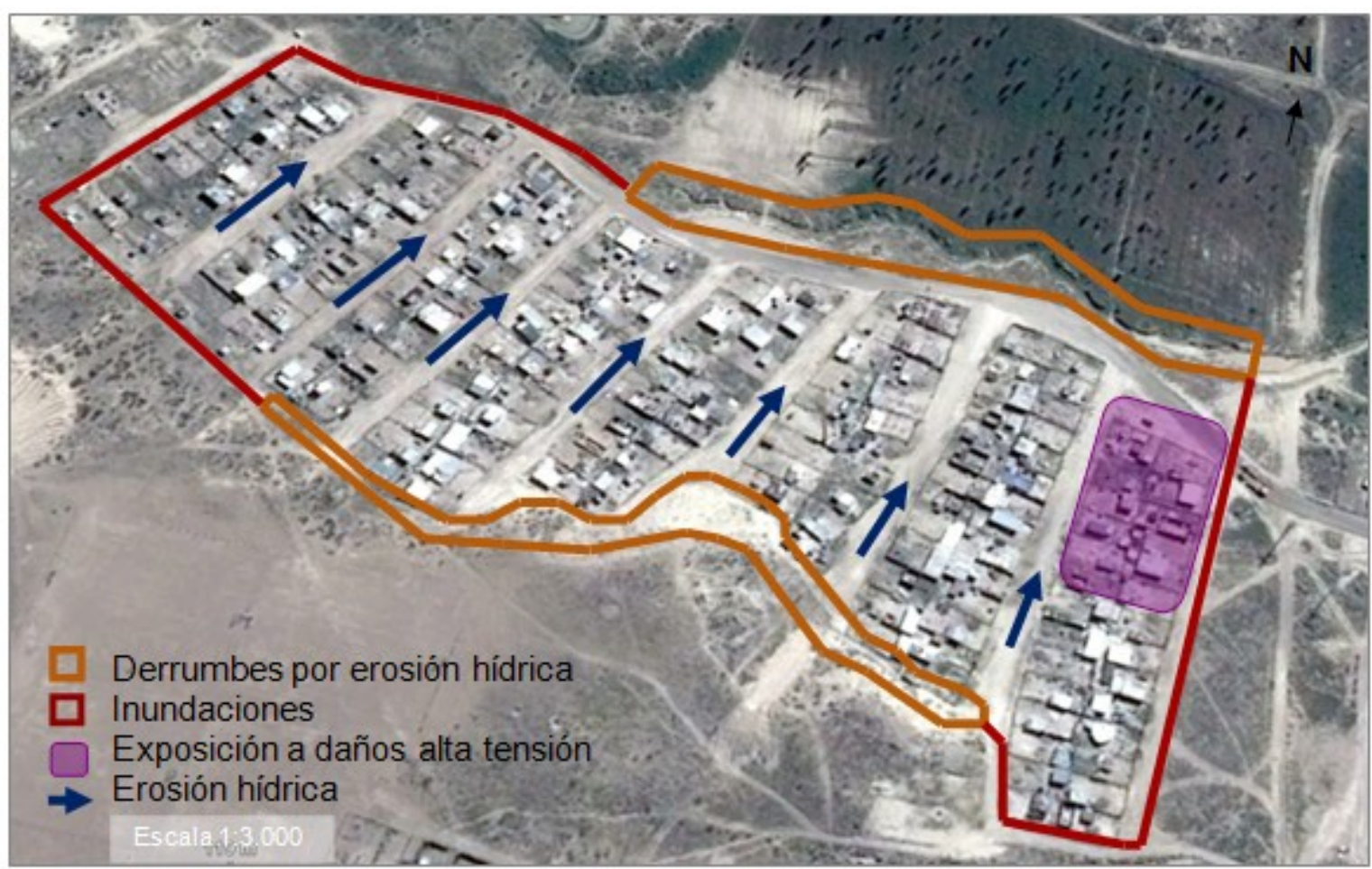

Fuente: elaboración propia sobre imagen Google Earth 2015

En el asentamiento La Lomita, los procesos de peligrosidad presentes son los siguientes:

- Derrumbes por erosión hídrica: estos procesos involucran a las viviendas que se localizan en 
el sector más elevado topográficamente, frente al espacio de esparcimiento "la bombonerita”. Corresponde a un proceso de peligrosidad natural inducida antrópicamente.

- Inundación y soterrado: este proceso de peligrosidad se produce a partir del material que es trasladado por el agua de lluvia que se desplaza desde el sector más elevado, buscando la pendiente natural. El mismo inunda y se deposita sobre las viviendas que se encuentran por debajo de la línea de calle en el sector este del asentamiento. Corresponde a un proceso de peligrosidad natural.

- Erosión hídrica: este tipo de proceso de peligrosidad de tipo natural se evidencia en la calle el Mirador, que es la que presenta mayor pendiente (ver figura 8). En momentos de precipitaciones extraordinarias se produce la erosión del sustrato.

\section{Figura 8}

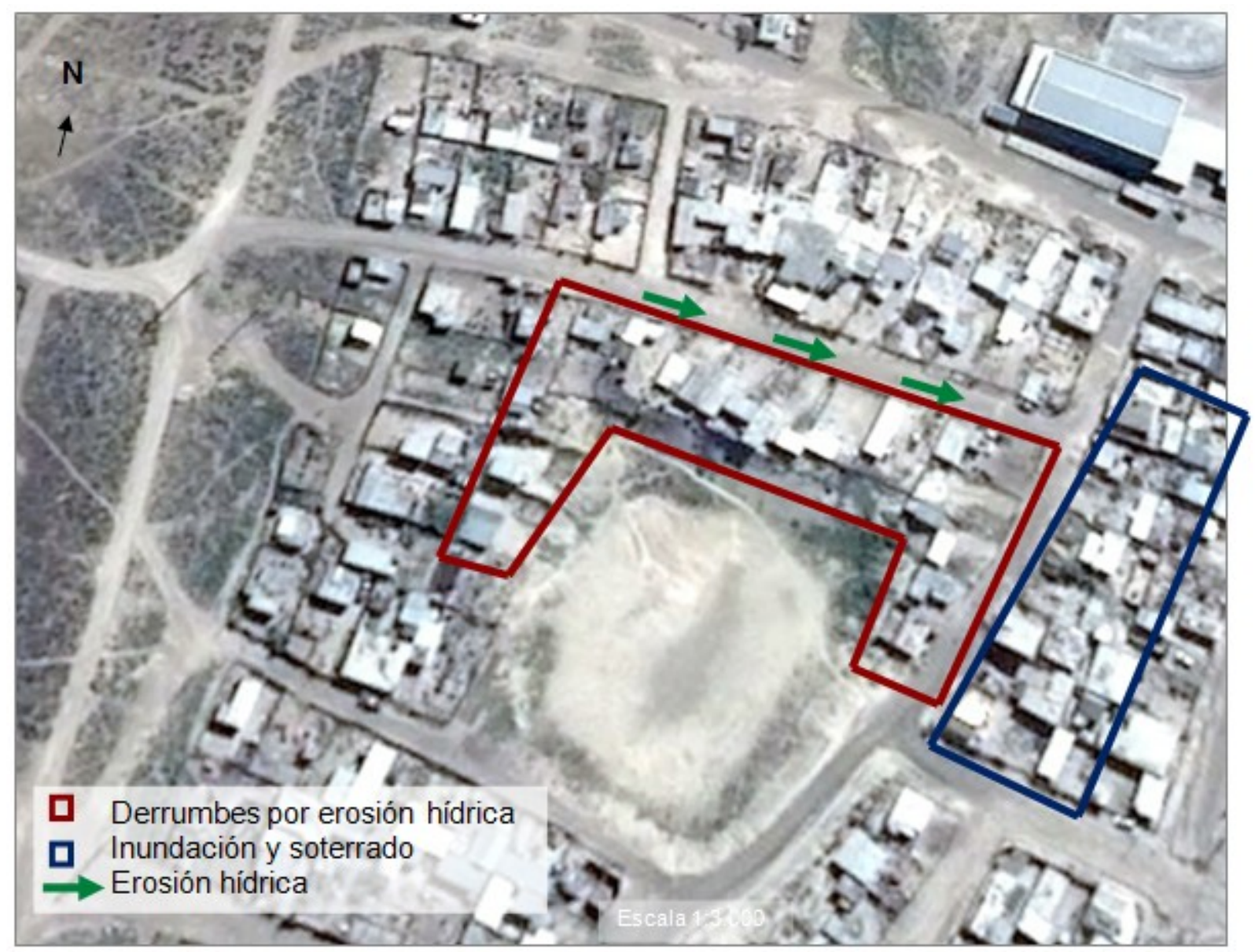

Fuente: elaboración propia sobre imagen Google Earth 2015

En cuanto a las condiciones de vulnerabilidad, se identifican los siguientes aspectos en ambos asentamientos: a) Vulnerabilidad física por localización: ambos asentamientos se localizan en sectores no aptos para la instalación humana, debido a las condiciones topográficas y a los procesos de peligrosidad allí presentes; b) Vulnerabilidad socioeconómica: la falta de acceso a servicios básicos -como es el agua, la electricidad y el gas-, sumado a la ausencia de transporte público, 
recolección de residuos, atención de la salud y vigilancia policial, son elementos que describen las condiciones precarias habitacionales de los residentes. Asimismo, los empleos que desarrollan la mayoría corresponden a actividades informales, changas, y en muchos casos se ha registrado la desocupación. Por lo tanto, estos elementos permiten afirmar que tanto el asentamiento La Lomita como Alta Tensión, presentan condiciones de vulnerabilidad en el aspecto social y el económico; c) Vulnerabilidad jurídica: los lotes ocupados por los vecinos no están mensurados y actualmente se encuentran en proceso de regularización de la tenencia de la tierra. A través del programa Promeba (Programa de Mejoramiento Barrial), el estado municipal y provincial están llevando adelante desde el año 2015 la regularización de tierras. Este proceso es lento, y sólo las familias con trabajo son quienes actualmente están pagando las cuotas de su terreno; muchas familias aún no pueden acceder a este beneficio.

\section{Percepción social del riesgo}

Los resultados del relevamiento de las percepciones del riesgo, mediante encuestas en ambos asentamientos de estudio, se presentan en los gráficos siguientes. Asimismo, de los talleres realizados pudo ampliarse la información referida tanto a las percepciones de los procesos de peligrosidad como a las condiciones de vulnerabilidad identificadas.

\subsection{Las percepciones del riesgo en el asentamiento La Lomita}

Respecto a los procesos de peligrosidad, mediante encuesta se indagó si los residentes perciben la probabilidad de derrumbes por erosión hídrica en aquellas viviendas localizadas en el sector más alto del asentamiento. En la figura 9 se observa que el $75 \%$ del total de personas encuestadas considera que el sector más elevado no se encuentra en un sitio seguro. Al indagar las causas, varias personas manifestaron lo siguiente: "cuando llueve el agua baja de golpe y arrasa, hubo una casa que se dañó mucho por eso”; “El sector más inseguro es el Pasaje El Mirador, tiene mucha pendiente y cuando llueve el agua baja de golpe e inunda las casas que están más bajas”.

\section{Figura 9}

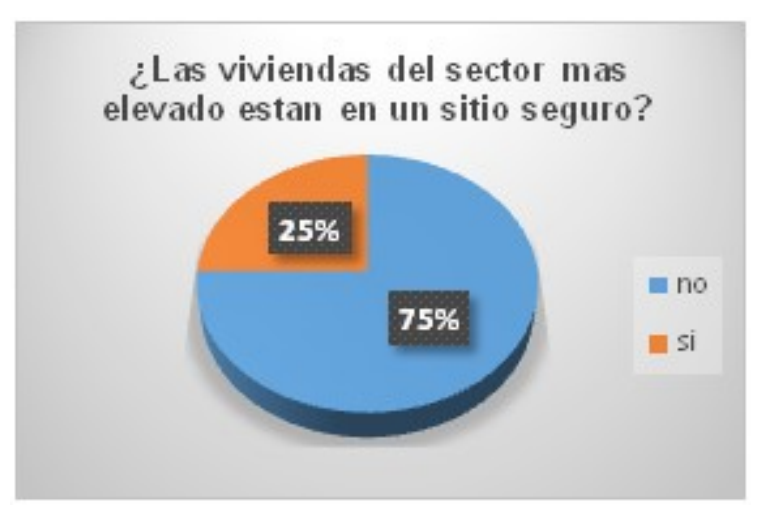

Fuente: elaboración propia 


\section{$\underline{\text { Figura } 10}$}

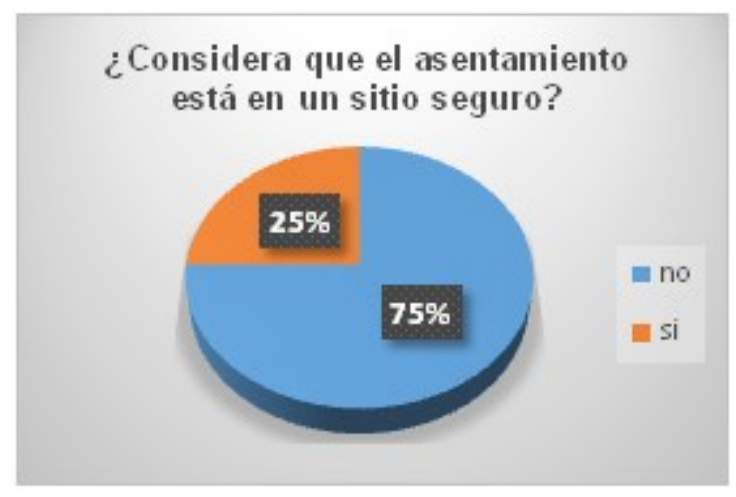

Fuente: elaboración propia

En la figura 10 se observa que el 75\% de los encuestados considera que el asentamiento La Lomita se localiza en un sitio inseguro, manifestando que cuando llueve hay viviendas que se inundan; es decir, que la condición de vulnerabilidad física por localización es percibida por la mayoría de los residentes. También, varias personas encuestadas manifestaron como inseguridad la frecuencia de robos en las viviendas e incendios por tomas clandestinas de electricidad.

Cuando se indagó sobre la percepción de las obras que aún faltan concretar en el asentamiento, el 100\% respondió que su ausencia es un problema urgente a resolver (ver figura 11), destacando que son sumamente necesarias sobre todo las obras de electricidad y gas (perciben su condición de vulnerabilidad socioeconómica). Respecto a la titularidad de los terrenos, el $100 \%$ de los encuestados manifestó su urgencia y necesidad, explicitando en muchos casos lo siguiente al respecto: "porque uno tiene algo que les es propio" (ver figura 12).

\section{Figura 11}

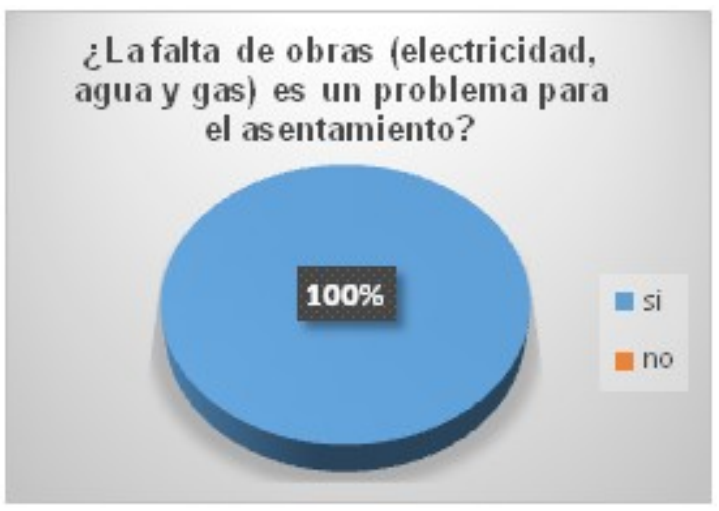

Fuente: elaboración propia 


\section{Figura 12}

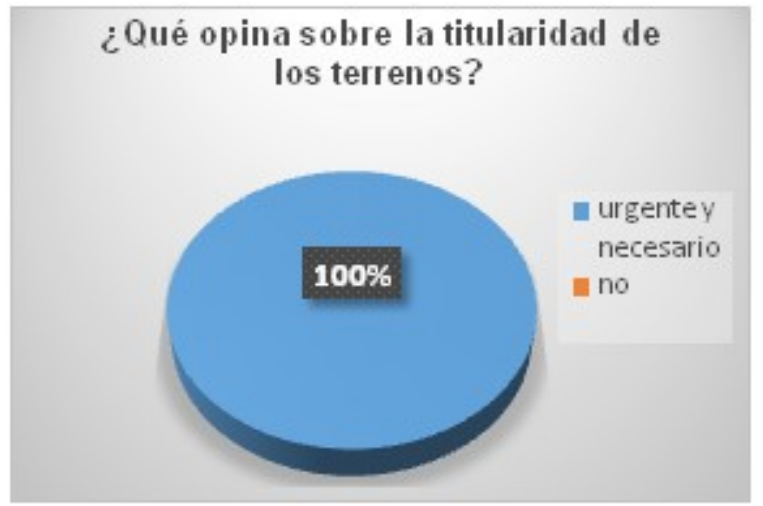

Fuente: elaboración propia

\subsection{Las percepciones del riesgo en el asentamiento Alta Tensión}

En el asentamiento Alta Tensión el estudio de las percepciones se focalizó tanto en aquellos procesos de peligrosidad natural -derrumbes por erosión hídrica, erosión hídrica en las calles, inundaciones- como la probabilidad de daños por la proximidad a la línea de alta tensión municipal (proceso de peligrosidad de tipo tecnológico). Los resultados de las encuestas muestran que el 83\% de las personas encuestadas considera que las viviendas ubicadas sobre laderas y próximas a cañadones no se encuentran en un sitio seguro (ver figura 13), expresando que cuando llueve el suelo es erosionado y suelen ocurrir derrumbes y daños en las viviendas. En la figura 14 se observa que el 95\% de los encuestados considera que las viviendas que se encuentran próximas a la línea de alta tensión no están en un sitio seguro, por posibles daños que pueden ocurrir a las personas y los bienes.

\section{Figura 13}

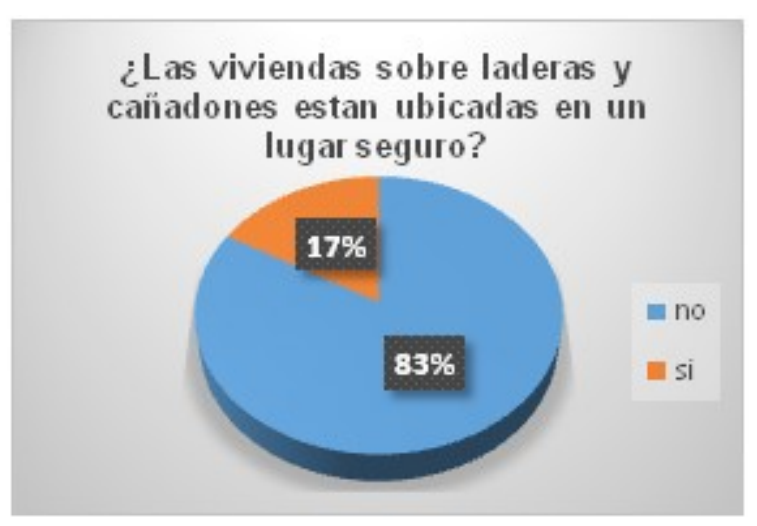

Fuente: elaboración propia 


\section{Figura 14}

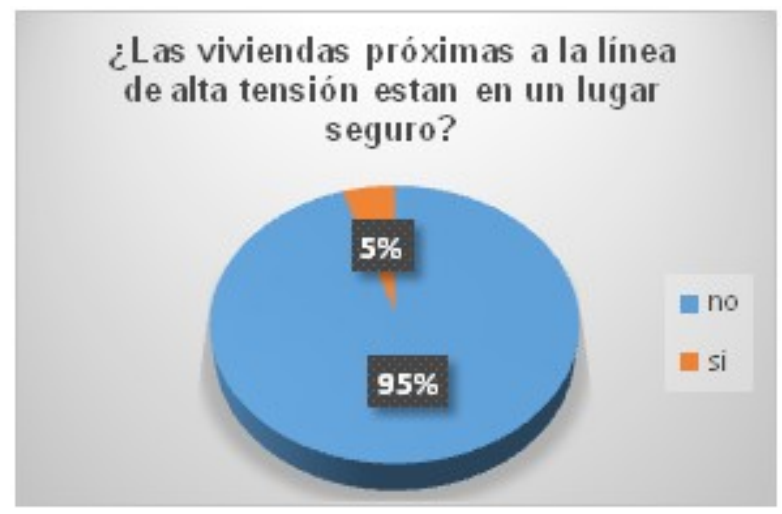

Fuente: elaboración propia

Respecto a la percepción de las condiciones de vulnerabilidad, el 97\% considera que el asentamiento Alta Tensión no se ubica en un sitio seguro. Este hecho pone de manifiesto la percepción por parte de los asentados de la condición de vulnerabilidad física por localización (ver figura 15). Asimismo, la falta de obras (electricidad, agua y gas) es percibida como un problema grave para el asentamiento, a resolver de manera urgente (ver figura 16).

\section{$\underline{\text { Figura } 15}$}

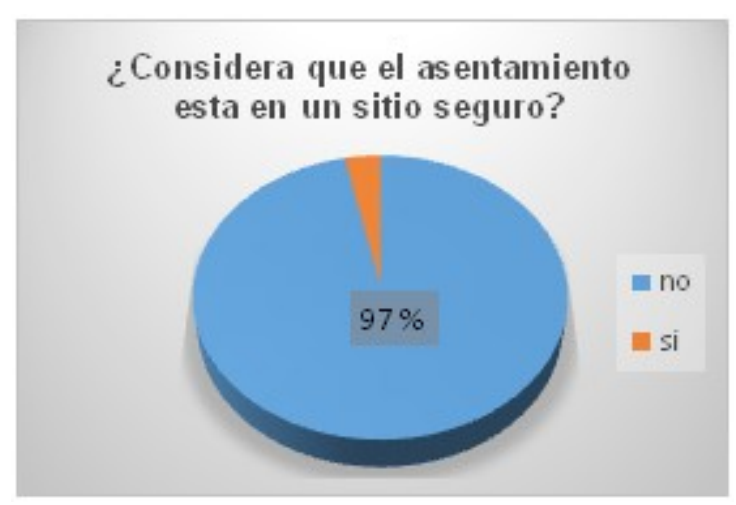

Fuente: elaboración propia

\section{$\underline{\text { Figura } 16}$}

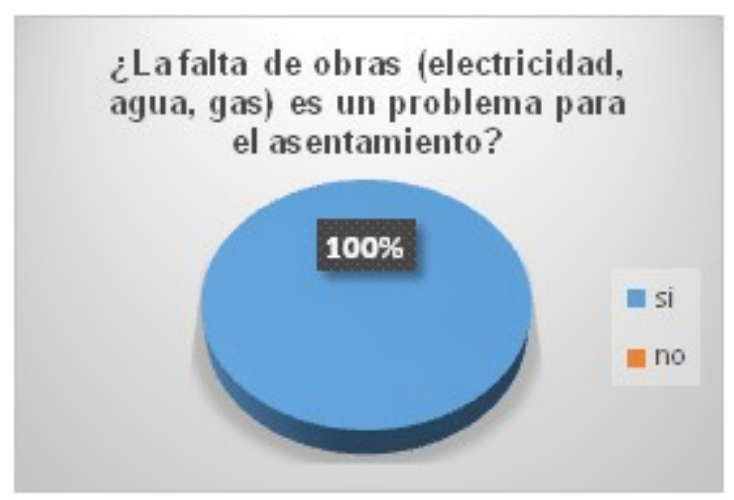

Fuente: elaboración propia 
Sobre la percepción de vulnerabilidad jurídica, el 92\% de las personas encuestadas percibe la necesidad urgente de acceder a la titularidad de los terrenos. En el asentamiento Alta Tensión el 92\% ya está pagando su terreno en cuotas al Municipio (figura 17).

\section{Figura 17}

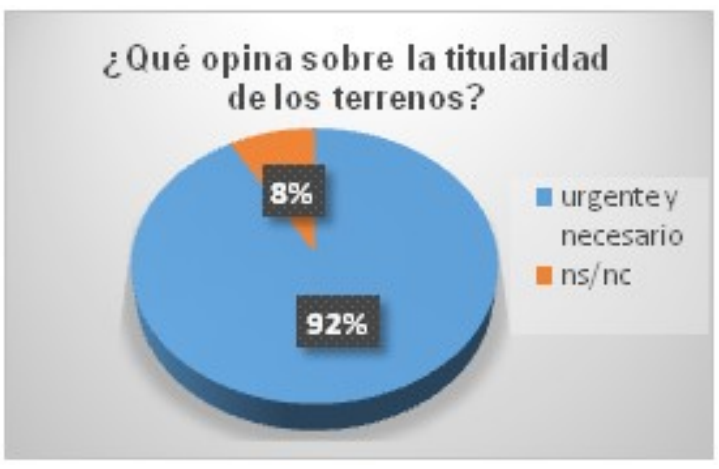

Fuente: elaboración propia

\section{Reflexiones finales}

Abordar dos diagnósticos sobre los asentamientos estudiados, es decir, uno tendiente a identificar las condiciones de riesgo presentes -con sus dos componentes principales: peligros y vulnerabilidades-, y el otro focalizado en las percepciones de los propios habitantes respecto a esta problemática, fue planteada con la finalidad de poder realizar comparaciones sobre los resultados obtenidos y así identificar la existencia de similitudes y/o posibles diferencias, tendientes a proponer soluciones y/o propuestas de gestión.

Se identificaron procesos de peligrosidad natural e inducidos antrópicamente en el asentamiento La Lomita. Similar situación ocurre en el asentamiento Alta Tensión, sumado a procesos de peligrosidad tecnológica. Estos mismos aspectos son percibidos como procesos de peligrosidad por los residentes de ambos sitios. Por otro lado, se identificaron ciertos aspectos vinculados a las condiciones de vulnerabilidad en ambos asentamientos, del tipo socioeconómico, jurídico y aquel relacionado con las condiciones de localización y emplazamiento (vulnerabilidad física por localización). Al igual que en las peligrosidades, estas variables de vulnerabilidad también son percibidas por los propios habitantes del lugar.

La gestión de riesgos es un complejo proceso social cuyo objetivo principal es disminuir las condiciones de riesgo en una sociedad, para lo cual debe intervenirse -a través de medidas, acciones o herramientas- la amenaza o peligro y la vulnerabilidad. La complejidad de tal proceso radica en que no sólo interviene el Estado como principal actor responsable en brindar soluciones, sino que además debe articularse con otros actores sociales, como ser las comunidades involucradas, las instituciones académicas o los medios de comunicación (Lavell y Arguello, 2003). Un proceso participativo de gestión del riesgo, implica que las decisiones no tienen por qué estar en manos exclusivamente de técnicos y especialistas, ni depender de un conocimiento superior; sino que en la toma de decisiones y en el desarrollo de las actuaciones adquieren relevancia los conocimientos de 
todos los actores sociales involucrados, incluyendo las comunidades que conviven con situaciones de riesgo.

Del proceso de comparación de los resultados obtenidos en los asentamientos La Lomita y Alta Tensión, se identifican similitudes entre los resultados del diagnóstico del riesgo y de las percepciones del riesgo. En el contexto de la gestión de riesgos, estas similitudes son consideradas como fortalezas al momento de proponer acciones o iniciativas para modificar la realidad problemática (므). La opinión de la comunidad afectada deber ser considerada al momento de brindar soluciones a problemas de este tipo, y el escenario se torna propicio cuando la propia comunidad percibe los diversos aspectos de la problemática que es diagnosticada. Como sostienen Prades López y González Reyes (1996, p. 3) "la percepción del riesgo debe entenderse como un instrumento para el desarrollo de políticas de prevención y gestión del riesgo”. En este sentido, se reconoce la importancia y la necesidad de incluir el imaginario del riesgo que las comunidades construyen por dos aspectos fundamentales: arribar a un diagnóstico integral y fomentar su articulación en los procesos de gestión.

\section{Notas}

1. Nueva Esperanza, La Arboleda, 27 de octubre, Nuevo Madryn y Nueva Chubut.

2. Las dimensiones física, socioeconómica y jurídica de vulnerabilidad son indagadas en esta investigación, tanto en el diagnóstico del riesgo como en las encuestas de percepción. Dichas categorías conceptuales fueron adaptadas a la realidad de los asentamientos en estudio.

3. Referente de Alta Tensión (L.B.), referente de La Lomita (E.Z.), referente Dirección Municipal de Tierras (Arq. M.F.) referente del centro de salud del Barrio Pujol (M.F.)

4. Se confeccionó una encuesta para el asentamiento La Lomita y otra para el asentamiento Alta Tensión, siguiendo los lineamientos de Ferrari (2015).

$\underline{5}$. Aquella en el que las unidades muestrales se escogen a partir de las referencias de las personas que van siendo encuestadas.

6. Por el contrario, las diferencias halladas entre el riesgo y las percepciones son consideradas debilidades sobre las cuales poner el foco en el proceso de gestión.

\section{Referencias}

Caneto, C. (2000). Geografía de la percepción. ¿Cómo vemos la ciudad? Buenos Aires: Lugar Editorial.

Cardona, O. D. (1993). Evaluación de la amenaza, la vulnerabilidad y el riesgo. Elementos para el ordenamiento y la planeación del desarrollo. En Maskrey, A. (ed), Los desastres no son naturales (pp. 51-73). Bogotá, Colombia: La Red de Estudios Sociales. 
Cardona, O. D. (2003). La necesidad de repensar de manera holística los conceptos de vulnerabilidad y riesgo. Una crítica y una revisión necesaria para la gestión. Recuperado de http://www.desenredando.org, Bogotá, Colombia: Centro de estudios sobre desastres y riesgos. Universidad de los Andes.

Ferrari, M. P. (2015). Aportes teóricos y metodológicos para el estudio de la percepción social de riesgos. Párrafos Geográficos, 14(2), 8-28.

Ferrari, M. P. y Bozzano, H. (2016). Aplicación del método Territorii en dos asentamientos espontáneos de Puerto Madryn, Argentina. Papeles de Geografía, 62, 137-151.

Guerrero, J. L. (2015). Densificación residencial informal en barrios y colonias del Área Metropolitana de Guadalajara. Dilemas y modelos espaciales. En Vidal-Koppmann, S. (comp.), Metrópolis en Mutación (pp. 239-265). Buenos Aires: Editorial Café de las Ciudades.

Lavell, A. y Arguello, M. (2003). Gestión de riesgos: un enfoque prospectivo. Colección Cuadernos de Prospectiva, 3, 37-46.

Monti, A. (2011). La peligrosidad de sitio en escenarios de riesgos: una propuesta de clasificación integral. Párrafos Geográficos, 10(2), 22-37.

Prades López, A. y González Reyes, F. (1996). La percepción social del riesgo: algo más que discrepancias entre expertos y público. En Prades López, A. (comp.), Energía, tecnología y sociedad (pp. 42-57). Madrid, España: Ediciones Torre.

Puy Rodríguez, A. (2002). Percepción social del riesgo: dimensiones de evaluación y predicción. Recuperado de: http://eprint.ucm.es/tesis/19911996/S/4/S4007501.pdf

Wilches Chaux, G. (1993). La vulnerabilidad global. En Maskrey, A. (comp.), Los desastres no son naturales (pp. 9-50). Bogotá, Colombia: La Red de Estudios Sociales. 